MS. MARJAANA JENNI JASMIINA HÄYRINEN (Orcid ID : 0000-0002-6801-5271)

Article type : Letter to Editor

\title{
Twist and Zeb1 expression identify Mycosis fungoides patients with low risk of disease progression
}

Marjaana J. Häyrinen ${ }^{1,2^{*}}$ and Pyry M. Uotila ${ }^{1,2}$, Helka Sahi ${ }^{3}$, Kirsi-Maria Haapasaari4 Hanna-Riikka Teppo ${ }^{4}$, Ylermi Soini ${ }^{5}$, Maria Lapela6 ${ }^{6}$ Kaija Vasala7, Taina Turpeenniemi-Hujanen ${ }^{1,2}$, Annamari Ranki ${ }^{3}$, Milla E. L. Kuusisto ${ }^{1,8}$ and Outi Kuittinen ${ }^{1,2,9}$

1 Department of Oncology and Radiotherapy, Oulu University Hospital and University of Oulu, Oulu, 90220, Finland

2 Medical Research Center, Oulu University Hospital and Unit of Cancer and Translational Medicine Research, University of Oulu, Oulu, 90220, Finland

3 Department of Dermatology, Allergology and Venereology, University of Helsinki and Helsinki University Hospital, P.O. Box 160, 00029 HUS, Helsinki, Finland

${ }^{4}$ Cancer Research and Translational Medicine Research Unit, University of Oulu, Department of Pathology, Oulu University Hospital Oulu, 90220, Finland

This article has been accepted for publication and undergone full peer review but has not been through the copyediting, typesetting, pagination and proofreading process, which may lead to differences between this version and the Version of Record. Please cite this article as doi: 10.1111/JDV.16009

This article is protected by copyright. All rights reserved 
${ }^{5}$ Department of Clinical Pathology and Forensic Medicine, Cancer Center of Eastern Finland, Kuopio University Hospital and University of Eastern Finland, Kuopio, 70210, Finland

${ }^{6}$ Department of Oncology and Radiotherapy, Turku University Hospital and University of Turku, Turku, 20521, Finland

${ }^{7}$ Department of Oncology, Jyväskylä Central Hospital, Jyväskylä, 40620, Finland

${ }^{8}$ Department of Haematology, Oulu University Hospital and University of Oulu, Oulu, 90220, Finland

${ }^{9}$ Department of Oncology and Radiotherapy, University of Eastern Finland, Faculty of Health Medicine, Institute of Clinical Medicine, Kuopio University Hospital

*Corresponding author: Peltolankaari 20 a 33, 90230 Oulu, Finland; Phone: +358504125521; Fax: +358 83153229; email: marjaana.hayrinen@student.oulu.fi

Count words: 599 (excluding reference list), figures: 2

Key words: Cutaneous T-cell lymphoma, Mycosis Fungoides, epithelial-tomesenchymal transition, Twist, Zeb1, Slug

Running title: EMT-inducing TFs Twist, Zeb1 and Slug in cutaneous T-cell lymphomas

Funding: Research grants from Finnish Cancer Foundation (AR), Helsinki University Hospital Research Funds (AR) and Väisänen Fund in Terttu Foundation (TT-H).

Competing or conflicts of interests disclosures: Corresponding author and all coauthors: None. All forms are sent via ScholarOne Manuscripts.

Mycosis fungoides (MF) is the most common cutaneous T-cell lymphoma ${ }^{1}$. The clinical course is usually slow and the initial treatment is a skin-directed therapy ${ }^{2,3}$. When MF becomes refractory, systemic therapies are needed ${ }^{2,3}$. Disease progression is seen in 
about $30 \%$ of MF patients but no undisputable markers are available to identify these patients ${ }^{2,3}$. Twist, Zeb1 and Slug are transcription factors (TFs) that induce epithelial-tomesenchymal transition (EMT) and regulate metastasis and other detrimental steps in cancer progression ${ }^{4}$. Twist, a T-cell oncoprotein, is generally not expressed in mature lymphoid cells ${ }^{5,6}$. Zeb1 suppresses haematopoiesis and downregulates CD4 expression during T-cell maturation ${ }^{7}$. These TFs have received increasing interest in lymphoma pathogenesis as well. Previous studies have demonstrated increased Twist expression in advanced MF lesions, and deletions and mutations targeting the ZEB1 gene in MF-9. Sézary syndrome patients with biallelic ZEB1 gene inactivation have a poor prognosis $^{10}$. Our hypothesis is that immunohistochemical (IHC) detection of these TFs has potential prognostic value in MF.

In this study, the expression of Twist, Zeb1 and Slug proteins was studied in diagnostic MF biopsy specimens ( $n=39$ ) using IHC (Fig. 1). The median patient age was 61 years (range 29-86) and the median follow-up time was 61 months (range 8-164). Depending on the clinical setting, patients received skin-directed therapies (topical corticosteroids, phototherapy using psoralens and ultraviolet-A irradiation, ultraviolet-B (UVB) or narrowband-UVB, or total skin electron beam therapy (TSEB)), systemic therapies (including bexarotene, prednisolone, methotrexate and interferon- $\alpha$ ) or chemotherapy (including CHOP-type treatments) ${ }^{2,3}$. The $\mathrm{IHC}$ results were retrospectively compared with clinical parameters by statistical analysis. We chose two surrogate variables to indicate disease progression (clinician's decision): Initiation of systemic therapy or TSEB (time to next systemic treatment or TSEB; TTNST) or initiation of chemotherapy (time to next chemotherapy treatment; TTNCT). At ten years of follow-up, $78 \%$ of cases with high Twist expression in the diagnostic skin biopsy had received chemotherapy versus $27 \%$ of cases with low Twist expression ( $p=0.004$ ) (Fig. 2a). At ten years of follow-up, $15 \%$ of cases with high Zeb1 expression required systemic therapy or TSEB versus $54 \%$ of cases with low Zeb1 expression $(p=0.046)$ (Fig. 2b). The Twist- $/ Z e b+$ variable identified patients who responded to, and did well with, skin-directed therapies alone. Conversely, $60 \%$ of 
Twist $+/$ Zeb- cases had disease progression within four years $(p=0.044)$ (Fig. $2 c)$. Slug expression had no significant associations.

We propose that lesional expression of Twist and Zeb1 has potential prognostic value in MF. Immunohistochemical detection of these EMT-inducing TFs can delineate different prognostic groups among MF patients and could, for example, aid in selecting patients for allogenic stem cell transplantation. This is the first study to describe these associations between IHC-detected Zeb1 protein expression and the clinical course of MF. In line with previous findings ${ }^{7-10}$, Zeb1 protein expression was associated with a favorable disease outcome. MF cases with high nuclear Zeb1 expression had longer TTNSTs and these lymphomas were efficiently kept in check with topical steroids and UV-therapy alone. Since Zeb1 and Twist immunostaining is a rapid, technically simple and easily reproducible method, it is well suited for routine clinical use and has potential for prognostication in MF. Our study had several limitations. The sample size was relatively small, and we used novel time variables that may be affected by physician- or patientrelated factors. Genomic data was not available to be compared with the IHC results. However, responses to standard systemic therapies in MF are often short and relapses frequent. There is an unmet medical need to find more reliable prognostic markers and targeted therapies. In the future, molecular inhibitors of Twist or Zeb1 mimetics may be suitable options for targeted therapy of MF.

\section{Reference list}

1. Willemze R, Jaffe ES, Cerroni L, et al. Review article WHO-EORTC classification for cutaneous lymphomas. Blood 2019;105(10):3768-85.

2. Trautinger F, Eder J, Assaf C, et al. European Organisation for Research and Treatment of Cancer consensus recommendations for the treatment of mycosis fungoides/Sézary syndrome - Update 2017. Eur J Cancer 2017;77:57-74.

3. Klemke CD, Booken N, Weiss C, et al. Histopathological and immunophenotypical criteria for the diagnosis of Sézary syndrome in differentiation from other erythrodermic 
skin diseases: A European Organisation for Research and Treatment of Cancer (EORTC) Cutaneous Lymphoma Task Force Study of 97 cases. $\mathrm{Br} \mathrm{J}$ Dermatol 2015;173(1):93-105.

4. Lemma S, Karihtala P, Haapasaari KM, et al. Biological roles and prognostic values of the epithelial-mesenchymal transition-mediating transcription factors Twist, ZEB1 and Slug in diffuse large B-cell lymphoma. Histopathology 2013;62(2):326-33.

5. Arthur A, Cakouros D, Cooper L, et al. Twist-1 Enhances Bone Marrow Mesenchymal Stromal Cell Support of Hematopoiesis by Modulating CXCL12 Expression. Stem Cells 2016;34(2):504-9.

6. Goswami M, Duvic M, Dougherty A, Ni X. Increased Twist expression in advanced stage of Mycosis fungoides and Sézary syndrome. J Cutan Pathol 2012;39(5):500-7.

7. Vandewalle $C$, Roy F Van, Berx G. Review The role of the ZEB family of transcription factors in development and disease. Cell Mol Life Sci 2009;66(5):773-87.

8. Choi J, Goh, G, Walradt T, et al. Genomic landscape of cutaneous T cell lymphoma. Nat Genet 2015;47(9):1011-19.

9. McGirt LY, Jia P, Baerenwald DA, et al. Whole-genome sequencing reveals oncogenic mutations in mycosis fungoides. Blood 2015;127:508-19.

10. Caprini E, Bresin A, Cristofoletti C, et al. Loss of the candidate tumor suppressor ZEB1 (TCF8, ZFHX1A) in Sézary syndrome. Cell Death Dis 2018; 9(12):1178. 

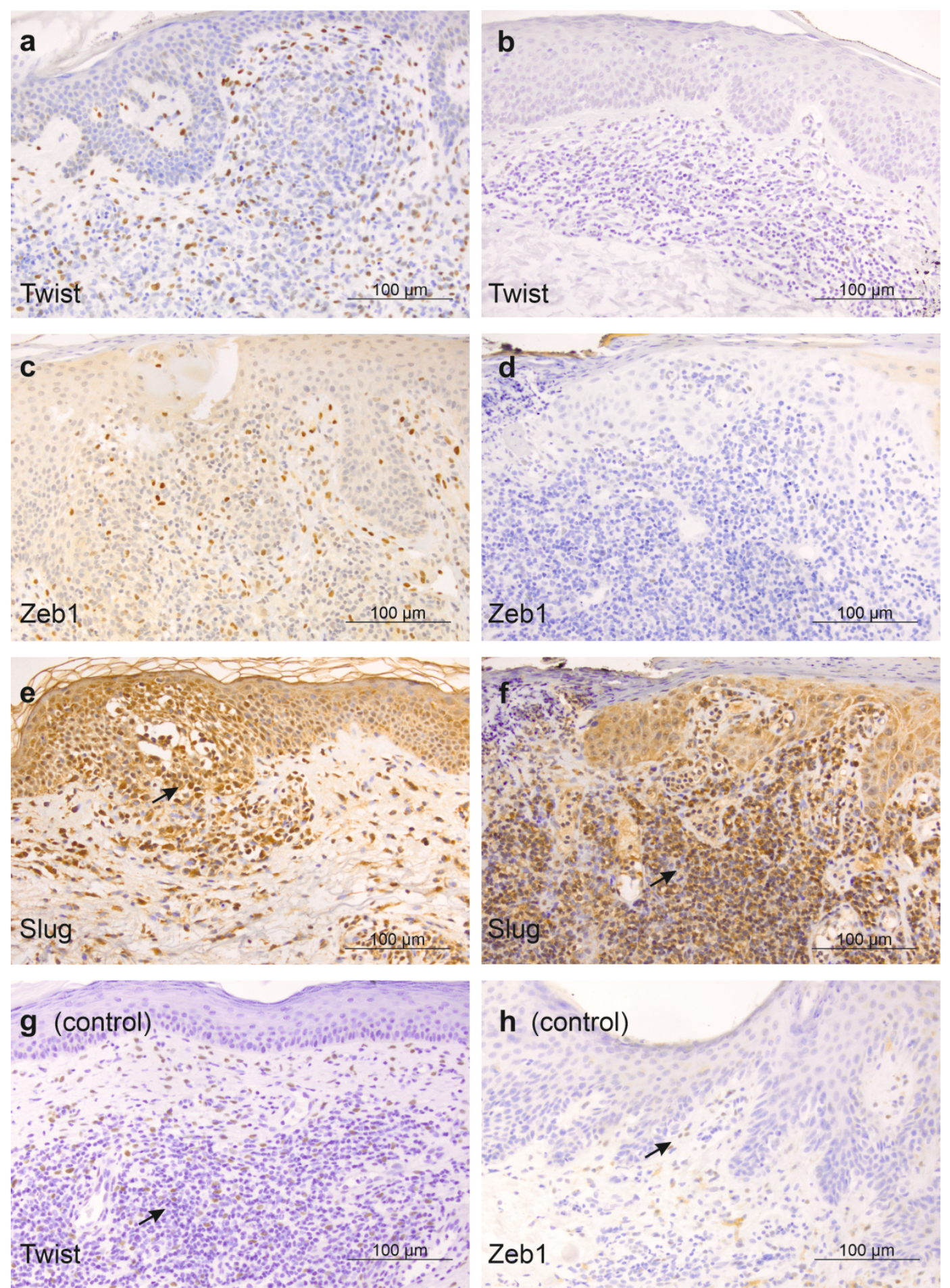

$$
\text { jdv_16009_f1.tif }
$$




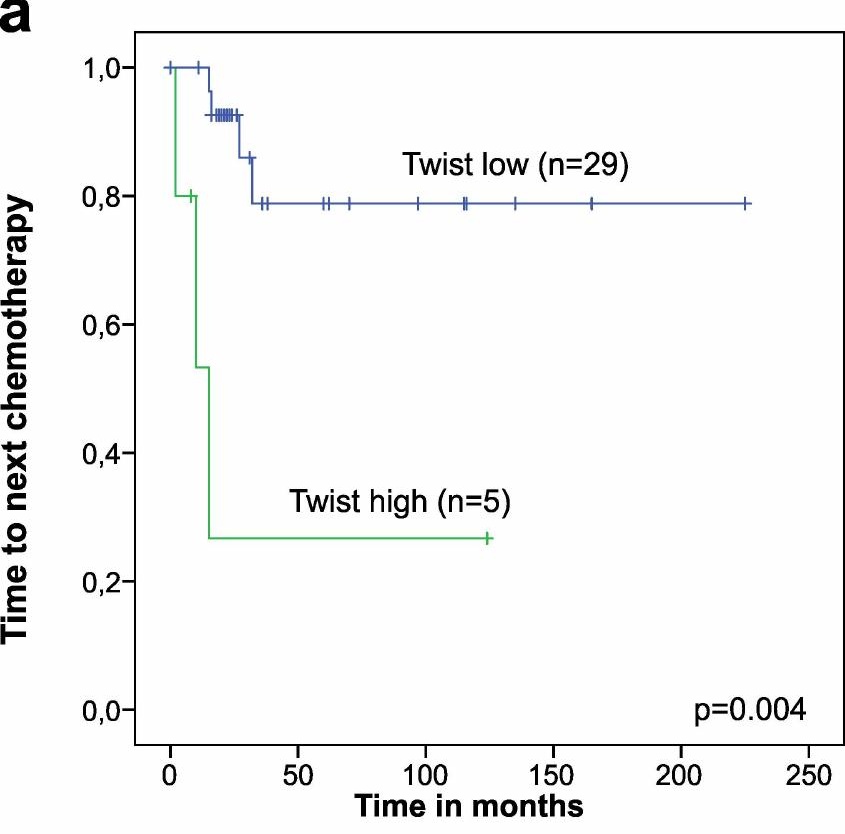

b
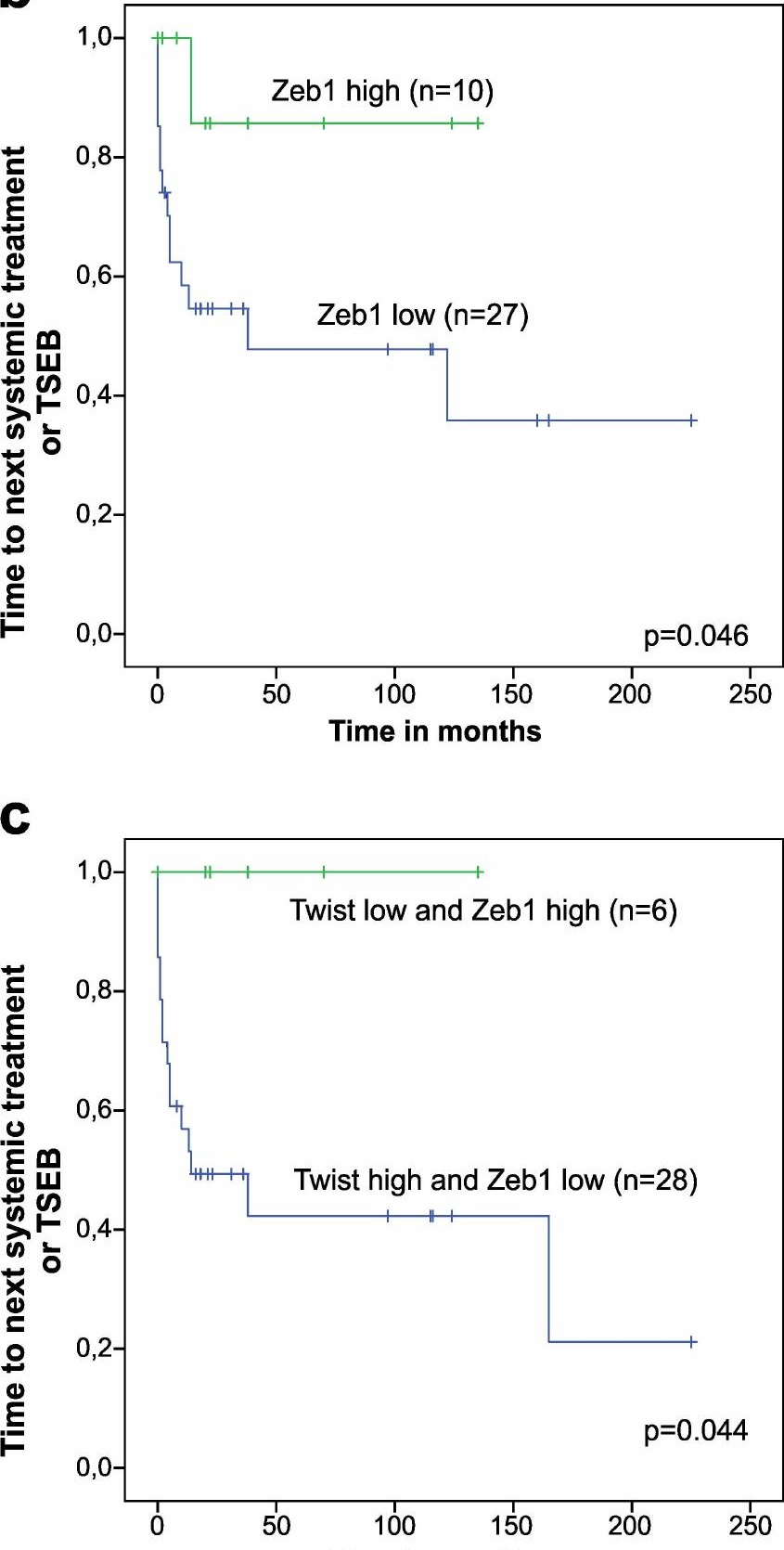